Gut and Liver, Vol. 9, No. 2, March 2015, pp. 167-173

\title{
Clinicopathologic Characteristics of Interval Gastric Cancer in Korea
}

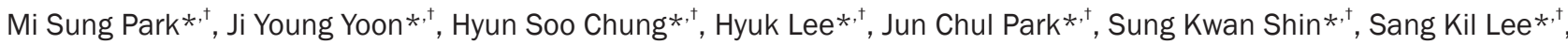 \\ and Yong Chan Lee*, \\ ${ }^{*}$ Department of Internal Medicine and ${ }^{\dagger}$ Institute of Gastroenterology, Yonsei University College of Medicine, Seoul, Korea
}

See editorial on page 133.

Background/Aims: Interval gastric cancer (IGC) is defined as cancer that is diagnosed between the time of screening and postscreening esophagogastroduodenoscopy (EGD). Unfortunately, little is known about the characteristics of IGC in Korea, a country with a high incidence of gastric cancer. The aim of this study was to evaluate the clinicopathologic characteristics of IGCs in Korea. Methods: From January 2006 to July 2011, a total of 81,762 subjects underwent screening EGD at Yonsei University Health Promotion Center, Seoul, Korea. We defined missed cancer as cancer diagnosed within 1 year of screening EGD and latent cancer as cancer diagnosed more than 1 year after EGD. Results: A total of 16 IGC patients (17 lesions; three missed cancers and 14 latent cancers) were identified, with a mean age of 60.68 years and a mean interval time of 19.64 months. IGCs tended to be undifferentiated $(12 / 17,70.6 \%)$, located in the lower body of the stomach $(12 / 17,70.6 \%)$ and exhibited flat/depressed endoscopic morphology $(11 / 17,64.7 \%)$. The patients with missed cancer were generally younger than the patients with latent cancer (51.3 years vs 62.8 years, $p=0.037$ ), and the patients with undifferentiated cancer were significantly younger than those with differentiated cancer ( 57.0 years vs 68.8 years, $p=0.008$ ). Conclusions: IGCs tended to be undifferentiated, located in the lower body of the stomach, and exhibited flat/depressed endoscopic morphology. (Gut Liver, 2015;9:167-173)

Key Words: Interval gastric cancer; Missed gastric cancer; Latent gastric cancer; Histopathology

\section{INTRODUCTION}

Gastric cancer is one of the most common cancers worldwide, with approximately 989,600 new cases and 738,000 deaths per year. ${ }^{1}$ Gastric cancer incidence rates are highest in Eastern Europe, South America, and Eastern Asia, especially in China, Japan, and Korea. ${ }^{2,3}$ However, mortalities associated with gastric cancer have decreased remarkably in Asian countries in recent years, potentially due to healthcare policies that have introduced screening tests for the early detection of gastric cancer. ${ }^{4}$ Early detection of gastric cancer is important because the prognosis of early gastric cancer is highly favorable. ${ }^{5,6}$ For this reason, esophagogastroduodenoscopy (EGD), the diagnostic tool of choice for detecting gastric cancer, has been widely conducted as a part of health checkups in recent years.

In Korea, national gastric cancer screening was instituted in 1999 as part of the National Cancer Screening Program (NCSP). The NCSP recommends biennial gastric cancer screening for males and females older than 40 years of age, using direct or indirect upper gastrointestinal series (UGIS) or EGD. ${ }^{7}$ However, there is a debate about the quality of screening EGD and appropriate screening intervals, leading to the concept of interval cancer.

Interval cancer is defined as cancer that is diagnosed between the time of screening and postscreening endoscopy. ${ }^{8}$ Considering colorectal cancer, some consensus and quality indicators have recently been established, ${ }^{8,9}$ and show that improvements in the quality of colonoscopy may have reduced cancer prevalence or resulted in earlier cancer detection in over 50\% of prevalent cancers. ${ }^{9}$ However, little is known about the characteristics, incidence, and cause of interval gastric cancer (IGC), especially in a country with a high gastric cancer incidence.

In this study, we assessed the performance of endoscopic surveillance screening at a single qualified institute. The aim of this

\footnotetext{
Correspondence to: Yong Chan Lee

Department of Internal Medicine, Yonsei University College of Medicine, 50 Yonsei-ro, Seodaemun-gu, Seoul 120-752, Korea

Tel: +82-2-2228-1960, Fax: +82-2-393-6884, E-mail: leeyc@yuhs.ac

Received on November 15, 2013. Revised on January 8, 2014. Accepted on January 21, 2014. Published online on June 18, 2014

pISSN 1976-2283 eISSN 2005-1212 http://dx.doi.org/10.5009/gnl13425

Mi Sung Park and Ji Young Yoon contributed equally to this work as first authors.

@ This is an Open Access article distributed under the terms of the Creative Commons Attribution Non-Commercial License (http://creativecommons.org/licenses/by-nc/3.0) which permits unrestricted non-commercial use, distribution, and reproduction in any medium, provided the original work is properly cited.
} 
study was to evaluate the clinicopathologic characteristics of IGC in Korea and to propose quality indicators for EGD screening.

\section{MATERIALS AND METHODS}

\section{Study population}

Gastric cancer screening data was obtained from the Yonsei University Health Promotion Center, Seoul, Korea from January 2006 to July 2011. The database included patients' demographic characteristics and screening EGD results, used for retrospective analysis. We selected patients who were diagnosed with gastric cancer following a previously-performed screening EGD at the same center. The control group is recruited as who underwent screening EGD on the same day with IGC patients at the same center with twice the number of patients. We did not conduct age- and sex-matching for recruiting control group because to compare clinicopathologic characteristics of IGC patients and control group, which included age and sex, we randomly recruited control group who underwent screening EGD just before and after IGC patients by the same endoscopist.

The exclusion criteria were as follows: no previous history of screening EGD; previous history of screening EGD at outside our institute because we could not determine the exact results of screening EGD; and previous history of gastric cancer.

The study was performed in accordance with the ethical guidelines of the 1975 Declaration of Helsinki. Written informed consent was obtained from each participant or responsible family member after possible complications of the diagnostic procedures had been fully explained. This study was approved by the Institutional Review Board of Yonsei University.

\section{Esophagogastroduodenoscopy}

All EGDs were performed with a standard single-channel endoscope (GIF-Q260 or GIF-H260; Olympus Optical Co., Ltd., Tokyo, Japan). All six endoscopists were trained at single tertiary medical center before working at our institute and all of them were board-certified.

\section{Definitions and clinical analysis}

IGC is defined as gastric cancer which diagnosed between the time of screening and postscreening EGD at the same center. Missed cancer is defined as that diagnosed within 1 year of screening EGD and latent cancer as that diagnosed more than 1 year after screening EGD, based on a previous study. ${ }^{10}$ The following data were collected: demographic characteristics of study population, interval time between the time of diagnosis and screening EGD, cancer histology, and endoscopic findings.

We analyzed the IGC rate and the clinicopathologic characteristics of IGCs. We compared IGC patients and control group and compared latent and missed cancer. Additionally, comparing differentiated cancer with undifferentiated cancer based on histologic finding.

\section{Statistical analysis}

Descriptive data are presented as the percentage of patients or as the mean \pm standard deviation. The cumulative incidence rate of IGCs was calculated as the number of IGCs diagnosed per 10,000 endoscopic examinations. A chi-square test or Fisher exact test for categorical variables and an independent sample t-test or Mann-Whitney U-test for continuous variables was used. To compare continuous variables among groups, one-way analysis of variance (ANOVA) was used as appropriate. SPSS version 18.0 software (SPSS Inc., Chicago, IL, USA) was used for all statistical analyses. A p-value $<0.05$ on a two-tailed test was considered statistically significant.

\section{RESULTS}

\section{Study population characteristics}

During the study period, 81,762 subjects underwent EGD at our institute. Among them, 112 patients were diagnosed with gastric cancer. Of these, 96 patients were excluded according to our exclusion criteria. Finally, a total of 16 IGC patients (17

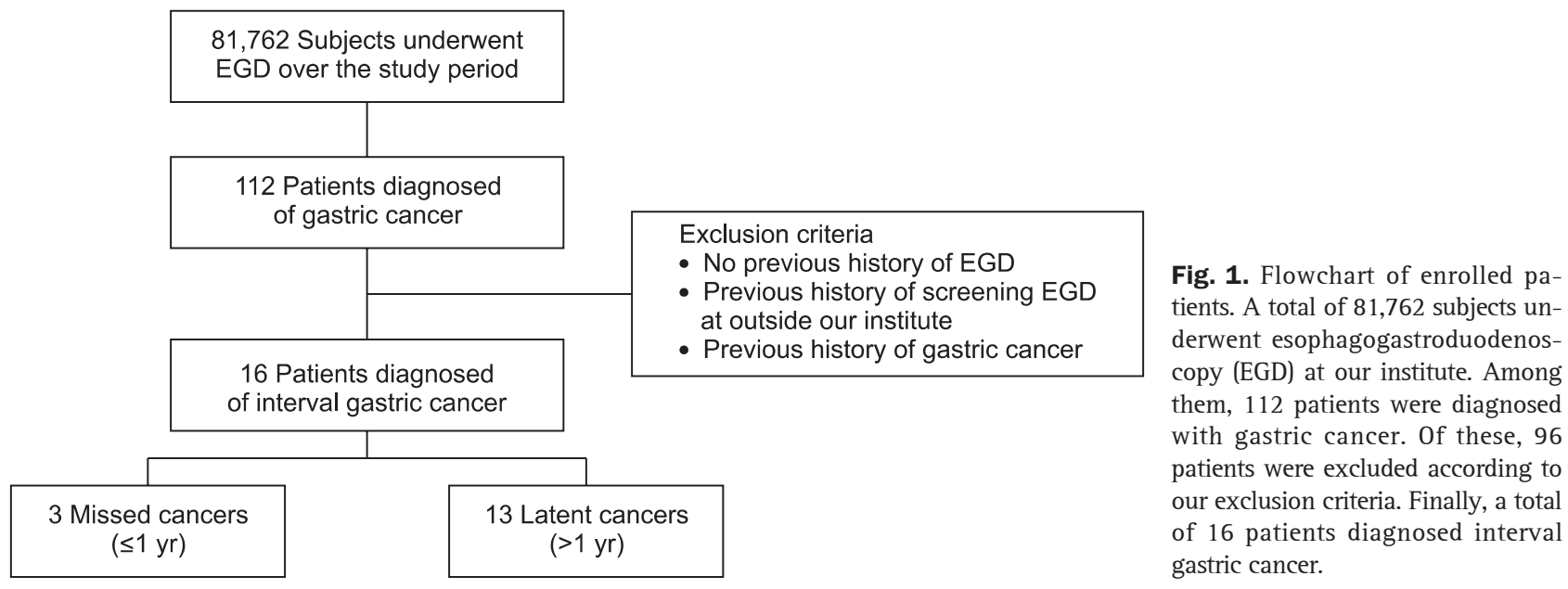


lesions) were identified (Fig. 1), 15 of 17 lesions were diagnosed as EGC and two of 17 as advanced gastric cancer (AGC).

Clinicopathologic characteristics of 16 patients (eight male) who diagnosed IGC are described in Table 1. The mean age was 60.68 years, and the mean interval time between the time of diagnosis and screening EGD was 19.64 months. One of IGC patient's screening and postscreening EGD are shown (Fig. 2). IGCs tended to be undifferentiated type (12/17, 70.6\%), located in lower body of stomach (12/17, 70.6\%), and have flat/de-

Table 1. Clinicopathologic Characteristics of the Interval Gastric Cancer Group ( $\mathrm{n}=16)$

\begin{tabular}{lr}
\hline \multicolumn{1}{c}{ Characteristic } & \multicolumn{1}{c}{ Value } \\
\hline Age, yr & $60.68 \pm 8.84$ \\
Male sex & $8(50.0)$ \\
Early gastric cancer & $15 / 17(88.2)$ \\
Advanced gastric cancer & $2 / 17(11.8)$ \\
Interval time, mo & $19.64 \pm 7.51$ \\
Macroscopic morphology & \\
Elevated & $6 / 17(35.3)$ \\
Flat & $5 / 17(29.4)$ \\
Depressed & $6 / 17(35.3)$ \\
Location I & \\
Lower body & $12 / 17(70.6)$ \\
Mid body & $3 / 17(17.6)$ \\
Upper body & $2 / 17(11.8)$ \\
Antrum & $0 / 17$ \\
Location II & \\
Anterior wall & $4 / 17(23.6)$ \\
Posterior wall & $5 / 17(29.4)$ \\
Lesser curvature & $3 / 17(17.6)$ \\
Greater curvature & $5 / 17(29.4)$ \\
Pathology & \\
Differentiated & \\
Undifferentiated & \\
\hline & \\
\hline
\end{tabular}

Data are presented as mean \pm SD or number $(\%)$. pressed endoscopic morphology (11/17, 64.7\%). The cumulative incidence rate IGCs was 2.07 per 10,000 patients, and most IGCs were detected at 12 to 18 months after screening EGD (Fig. 3).

\section{Comparison with control group}

In comparison with the control group, the IGC patients was significantly older (mean age, 60.7 years vs 53.5 years, $p=0.033$ ) (Table 2). There were no statistically significant differences about previous history of gastric ulcer, previous history of Helicobacter pylori infection, smoking status, alcohol ingestion, and family history of gastric cancer between two groups. In the screening EGD, a finding of atrophic gastritis was significantly frequent in the IGC patients than in the control group (52.9\% vs $9.4 \%, p=0.001$ ) but there were no significant differences in findings of intestinal metaplasia (IM), single erosion, and biopsy frequency.

\section{Previous situation of IGC lesion}

The previous situation of 17 IGC lesions based on their screening EGD results was described at Supplementary Table 1. Two lesions were atrophic gastritis and five were superficial gastritis without focal lesion. Three lesions were combined atrophic gastritis and diffuse IM. One lesion was combined atrophic gastritis and diffuse erosions and one lesion was combined atrophic gastritis and single erosion with pathologically confirmed. Two lesions were combined with atrophic gastritis with polypoid lesion, and IM was confirmed pathologically both of them. One lesion was combined with superficial gastritis and diffuse erosions, two lesions were combined superficial gastritis and single erosion, both of them pathologically confirmed with erosion.

\section{Comparison with latent cancer and missed cancer}

Among 17 lesions of IGC, three lesions (three patients) were missed cancers and 14 lesions (13 patients) were latent cancers. Patients with missed cancers were significantly younger than those with latent cancers (mean age, 51.3 years vs 62.8 years; $\mathrm{p}=0.037$ ), and although statistically not significant, latent cancer tended to more AGC than missed cancer (14.3\% vs 0\%, $\mathrm{p}>0.005$ ) (Table 3). All missed cancers were undifferentiated
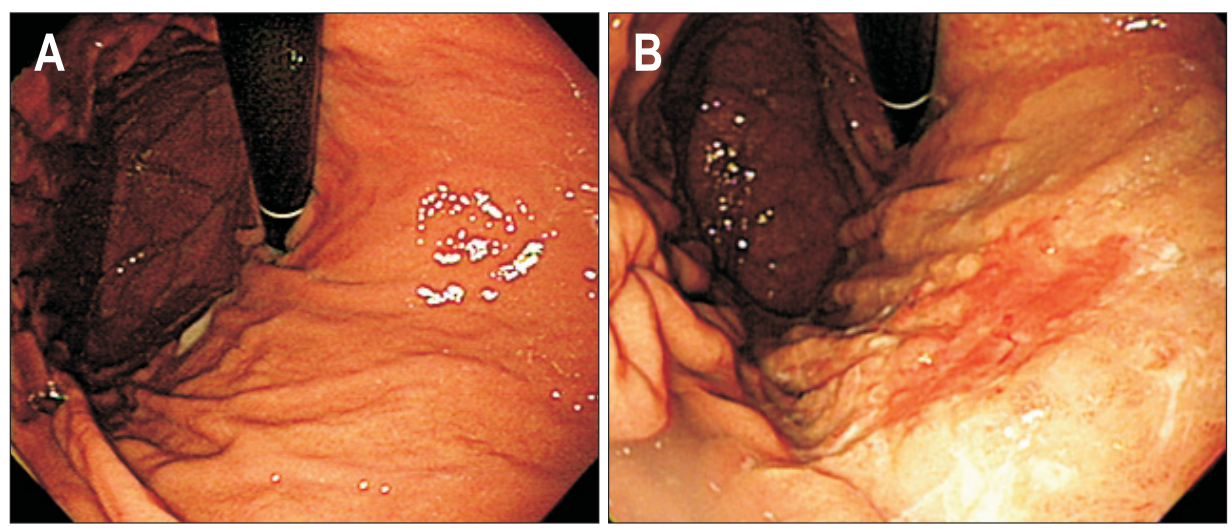

Fig. 2. Esophagogastroduodenoscopy (EGD) of an interval gastric cancer patient. A 50-year-old female's EGD at screening (A) and postscreening (B). Screening EGD finding was diffusely mild atrophic gastritis without focal lesion. However, after 15.6 months, early gastric cancer at the lower body, anterior wall of the stomach was diagnosed. Pathology was reported as signet ring cell carcinoma. 


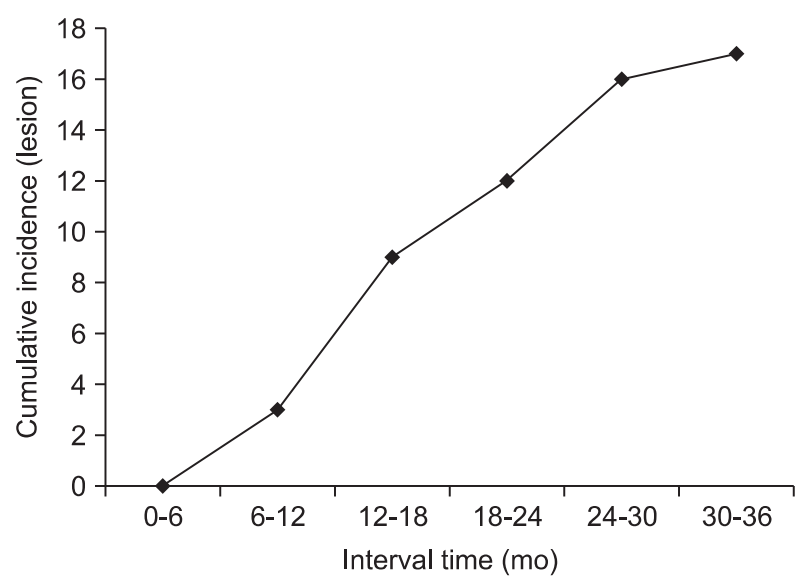

Fig. 3. Cumulative incidence of interval gastric cancer and interval time. The cumulative incidence rate of interval gastric cancer was 2.07 per 10,000 patients, and most incidences of interval gastric cancer were detected at 12 to 18 months after screening esophagogastroduodenoscopy (EGD).

Table 2. Comparison with the Control Group

\begin{tabular}{|c|c|c|c|}
\hline & $\begin{array}{l}\text { Interval cancer } \\
\text { group }(n=16)\end{array}$ & $\begin{array}{l}\text { Control group } \\
(\mathrm{n}=32)\end{array}$ & $\begin{array}{c}\mathrm{p}- \\
\text { value }\end{array}$ \\
\hline Age, yr & $60.68 \pm 8.84$ & $53.53 \pm 7.08$ & 0.033 \\
\hline Male sex & $8(50.0)$ & $18(56.3)$ & NS \\
\hline $\begin{array}{l}\text { Previous history of } \\
\text { gastric surgery }\end{array}$ & 0 & 0 & NS \\
\hline $\begin{array}{l}\text { Previous history of } \\
\text { gastric ulcer }\end{array}$ & $2(12.5)$ & $3(9.4)$ & NS \\
\hline $\begin{array}{l}\text { Previous history of } \\
\text { H. pylori infection }\end{array}$ & $4(25.0)$ & $7(21.9)$ & NS \\
\hline Smoking status & & & NS \\
\hline Current & $2(12.5)$ & $2(6.3)$ & \\
\hline Ex & $3(18.8)$ & $8(25.0)$ & \\
\hline None & $11(68.7)$ & $22(68.7)$ & \\
\hline Alcohol ingestion* & $2(12.5)$ & $5(15.6)$ & NS \\
\hline $\begin{array}{l}\text { Family history of } \\
\text { gastric cancer }\end{array}$ & $2(12.5)$ & $3(9.4)$ & NS \\
\hline Interval time, mo & $19.64 \pm 7.51$ & $20.93 \pm 8.71$ & NS \\
\hline \multicolumn{4}{|l|}{ Screening EGD } \\
\hline Procedure time, sec & $237 \pm 54.24$ & $231.84 \pm 88.01$ & NS \\
\hline Atrophic gastritis & $9(52.9)^{\dagger}$ & $3(9.4)$ & 0.001 \\
\hline Intestinal metaplasia & $3(17.6)^{\dagger}$ & $5(15.6)$ & NS \\
\hline Single erosion & $3(17.6)^{\dagger}$ & $4(12.5)$ & NS \\
\hline Biopsy frequency & $1.46 \pm 1.89$ & $1.04 \pm 1.55$ & NS \\
\hline
\end{tabular}

Data are presented as mean \pm SD or number $(\%)$.

NS, not significant; H. pylori, Helicobacter pylori; EGD, esophagogastroduodenoscopy.

*Alcohol ingestion in excess of $40 \mathrm{~g} /$ day for more than 3 years; ${ }^{\dagger} \%$ was calculated based on the number of lesions $(n=17)$.
Table 3. Comparison with Latent Cancer and Missed Cancer

\begin{tabular}{|c|c|c|c|}
\hline & Latent cancer & Missed cancer & $\begin{array}{c}\mathrm{p}- \\
\text { value }\end{array}$ \\
\hline Patient number (lesion) & $13(14)$ & $3(3)$ & \\
\hline Age, yr & $62.84 \pm 7.55$ & $51.33 \pm 9.07$ & 0.037 \\
\hline Male sex & $6(46.2)$ & $2(66.7)$ & NS \\
\hline Interval time, mo & $21.53 \pm 7.01$ & $11.67 \pm 0.57$ & 0.001 \\
\hline Early gastric cancer & $11(84.6)$ & $3(100.0)$ & NS \\
\hline Advanced gastric cancer & $2(15.4)$ & 0 & \\
\hline Macroscopic morphology & & & NS \\
\hline Elevated & $5(35.7)$ & $1(33.3)$ & \\
\hline Flat & $5(35.7)$ & 0 & \\
\hline Depressed & $4(28.6)$ & $2(66.7)$ & \\
\hline Location I & & & NS \\
\hline Lower body & $10(71.4)$ & $2(66.7)$ & \\
\hline Mid body & $2(14.3)$ & $1(33.3)$ & \\
\hline Upper body & $2(14.3)$ & 0 & \\
\hline Antrum & 0 & 0 & \\
\hline Location II & & & NS \\
\hline Anterior wall & $3(21.4)$ & $1(33.3)$ & \\
\hline Posterior wall & $5(35.7)$ & 0 & \\
\hline Lesser curvature & $2(14.3)$ & $1(33.3)$ & \\
\hline Greater curvature & $4(28.6)$ & $1(33.3)$ & \\
\hline Pathology & & & NS \\
\hline Differentiated & $5(35.7)$ & 0 & \\
\hline Undifferentiated & $9(64.3)$ & $3(100.0)$ & \\
\hline
\end{tabular}

Data are presented as mean \pm SD or number $(\%)$. NS, not significant.

type and 66.7\% (2 of 3) lesions showed depressive endoscopic morphology.

\section{Comparison with rapidly growing cancer and slowly growing cancer}

We further analyzed 17 IGC lesions as rapidly growing cancer and slowly growing cancer, according to interval time of 24 months which recommends by Korean NCSP7 (Supplementary Table 2). Five lesions (four patients) were slowly growing cancers and 12 lesions (12 patients) were rapidly growing cancers. Patients with rapidly growing cancer were tended to younger than those with slowly growing cancer without statistical significance (mean age, 58.7 years vs 66.2 years; $p>0.05$ ). There were no significant different in proportion of AGC, macroscopic morphology of cancer, location of cancer, and pathology in both two groups. 
Table 4. Comparison with Differentiated Cancer and Undifferentiated Cancer

\begin{tabular}{lccc}
\hline & $\begin{array}{c}\text { Undifferentiated } \\
\text { cancer }\end{array}$ & $\begin{array}{c}\text { Differentiated } \\
\text { cancer }\end{array}$ & p-value \\
\hline Patient number (lesion) & $11(12)$ & $5(5)$ & \\
Age, yr & $57.0 \pm 7.88$ & $68.8 \pm 4.26$ & 0.008 \\
Male sex & $6(54.5)$ & $2(40.0)$ & NS \\
Interval time, mo & $18.71 \pm 7.76$ & $21.25 \pm 7.25$ & NS \\
Early gastric cancer & $11(91.7)$ & $4(80.0)$ & NS \\
Advanced gastric cancer & $1(8.3)$ & $1(20.0)$ & \\
Macroscopic morphology & & & NS \\
Elevated & $4(33.3)$ & $2(40.0)$ & \\
Flat & $4(33.3)$ & $1(20.0)$ & \\
Depressed & $4(33.3)$ & $2(40.0)$ & \\
Location I & & & NS \\
Lower body & $8(66.7)$ & $4(80.0)$ & \\
Mid body & $3(25.0)$ & 0 & \\
Upper body & $1(8.3)$ & $1(20.0)$ & \\
Antrum & 0 & 0 & \\
Location II & & & \\
Anterior wall & $4(33.3)$ & 0 & \\
Posterior wall & $2(16.7)$ & $3(60.0)$ & \\
Lesser curvature & $2(16.7)$ & $1(20.0)$ & \\
Greater curvature & $4(33.3)$ & $1(20.0)$ & \\
\hline
\end{tabular}

Data are presented as mean \pm SD or number $(\%)$. NS, not significant.

\section{Comparison with differentiated cancer and undifferenti- ated cancer}

Based on histologic finding, 12 lesions were reported as undifferentiated cancer (11 patients) and five lesions (five patients) as differentiated cancer. Patients with undifferentiated cancer were significantly younger than patients with differentiated cancer (mean age, 57.0 years vs 68.8 years; $p=0.008$ ) (Table 4). Although statistically not significant, the mean interval time tended to be shorter in patients with undifferentiated cancer than in those with differentiated cancer (18.71 months vs 21.25 months, $\mathrm{p}>0.005$ ).

\section{Oncologic outcome of IGC patients}

Among of 16 IGC patients, two patients were lost to followup due to referral to outside institute at the time of cancer diagnosis. Among of 14 patients who treated and followed, five patients treated with endoscopic submucosal dissection and nine patients with gastrectomy. Thirteen patients (92.9\%) have lived with no disease recurrence until December 2013 (mean followup duration, 37.0 months), only one patient (7.1\%) experienced disease recurrence after gastrectomy with peritoneal seeding and now on chemotherapy. This patient's interval time was 33.4 months and cancer histology was signet ring cell carcinoma.

\section{DISCUSSION}

Screening EGD can be helpful for the early detection of gastric cancer. However, an optimal interval between screening tests has not been established due to the limited number of studies in this field.

In studies conducted in Japan, Shiratori et al. ${ }^{11}$ reported that a 1.5-year interval between screenings was sufficient to detect EGC. Mori et al. ${ }^{12}$ concluded that a 2-year screening interval was sufficient to increase the survival rate in gastric cancer. In Korean studies, Nam et al. ${ }^{13}$ showed that a 2-year endoscopic screening interval was helpful for detecting gastric cancers that warranted endoscopic resection. Lee et al. ${ }^{14}$ demonstrated that a maximal screening interval of 3 years is required to detect EGC in patients without IM, and a 2-year screening interval is recommended for patients with IM.

The Korean NCSP recommends biennial screening UGIS or EGD for older than 40 years of age. ${ }^{7}$ However, in this study, the mean interval time for IGCs was 19.64 months and most IGCs were detected at 12 to 18 months after screening, which is shorter than the 2-year interval recommended by the Korean NCSP. Additionally, especially in patients with undifferentiated cancer, which has poor prognosis, ${ }^{15,16}$ the interval time was shorter than those for patients with differentiated cancer. Overall, these results do not support the use of this 2-year guideline, and we are proposing reconsideration of the appropriateness of the 2-year screening interval.

Interval cancers can occur as a result of the failure to detect a mucosal abnormality at the time of screening or as a new event after a negative screening. ${ }^{17}$ Based on the gastric cancer doubling time of approximately 2 to 3 years ${ }^{18}$ and a mean interval time of 19.64 months of this study, IGCs in this study were considered likely due to failure of the screening EGD to detect an abnormality. And IGCs tended to exhibit flat/depressed endoscopic morphology (11/17, 64.7\%). The accuracy of endoscopic procedure is directly influenced by the skill of the endoscopists and/or the quality of endoscopic video systems and videoscope. ${ }^{19-21}$ To detect cancers with flat/depressed endoscopic morphology and to prevent failed attempts to detect a mucosal abnormality, the use of high-definition endoscopy equipment and performance of endoscopy by expert endoscopists are important.

This study utilized data obtained at a single qualified institute with similar quality of endoscopic video systems/videoscope and similary-trained endoscopists. The incidence rate of IGC in this study was $2.07 / 10,000$ as single qualified institute is meaningful in comparison of previously reported national institute data of that 1.25/1,000 with missed cancer in the first round and $1.11 / 1,000$ in the subsequent round. ${ }^{22}$ We think the reason of low miss rate in this study than previous study is due to 
qualified endoscopic device and endoscopist control of single institute. These results suggest the importance of standardized endoscopic video systems/videoscope and quality control among endoscopists in screening EGD.

In consideration of atrophic gastritis as a premalignant condition and the multifocal nature of the metaplastic process, ${ }^{23,24}$ thorough endoscopic evaluation is usually needed. ${ }^{25,26}$ Kato et $a{ }^{27}$ estimated that the risk of gastric cancer in individuals with atrophic gastritis was 25-fold for differentiated types and 3.5-fold for undifferentiated types of cancer. In this study, IGC patients showed significantly higher proportions of atrophic gastritis than the control group in their previous screening EGDs (52.9\% vs 9.4\%, $\mathrm{p}=0.001)$. This result suggests that patients diagnosed with atrophic gastritis in a screening EGD should be closely followed in their next EGD which coincide with previous study. ${ }^{23}$

In this study, IGCs tended to be more undifferentiated type, which has a poorer prognosis. ${ }^{15,16}$ And patients with undifferentiated type IGCs tended to be younger than those with differentiated cancers. Patients with missed cancer were younger than those with latent cancer and all three missed cancers were the undifferentiated type. Considering the poorer prognosis and younger age, there results raise the importance of detecting IGCs and missed cancers, which should be considered risky, especially in younger patients.

This study has some limitations. First, the retrospective study design and small sample size could cause statistical error or include confounding factors. Even if IM is regarded as another premalignant condition of gastric cancer, ${ }^{23,24}$ there was no significant difference finding of IM in screening EGD between the IGC patients and the control group. We think this might be due to small sample size. Second, selection bias in recruiting control group and IGC group who only underwent previous screening EGD at the same center and performed EGD by many endoscopists, because endoscopic finding is interpreted subjectively by endosopist, could weaken strength of this study. Third, this study design utilized a single qualified institute, so endoscopy/ endoscopist quality variables could not be evaluated. Further prospective large-scale designed study is needed to suggest IGCrelated risk factors and provide endoscopy/endoscopist quality indicators to predict early cancers based on results from this study.

However, recent publications ${ }^{8,9}$ on assessing rates of colorectal cancer missed during colonoscopy have generated efforts toward the improvement of colonoscopy quality, and this study could support that EGD quality control is important to enhance cancer detection rates and reduce IGC rates. We think the important factor for complete quality control of gastric cancer is endoscopy/endoscopist quality control.

In conclusion, IGCs tended to be undifferentiated, exhibit flat/ depressed endoscopic morphology, and located in the lower body of the stomach with shorter interval time than 2-year after screening EGD. The 2-year screening interval should be reconsidered and national endoscopy/endoscopist quality control is needed in diagnosing gastric cancers in Korea, a country with a high gastric cancer incidence.

\section{CONFLICTS OF INTEREST}

No potential conflict of interest relevant to this article was reported.

\section{REFERENCES}

1. Jemal A, Bray F, Center MM, Ferlay J, Ward E, Forman D. Global cancer statistics. CA Cancer J Clin 2011;61:69-90.

2. Siegel R, Ward E, Brawley O, Jemal A. Cancer statistics, 2011: the impact of eliminating socioeconomic and racial disparities on premature cancer deaths. CA Cancer J Clin 2011;61:212-236.

3. Parkin DM, Bray F, Ferlay J, Pisani P. Global cancer statistics, 2002. CA Cancer J Clin 2005;55:74-108.

4. Mizoue T, Yoshimura T, Tokui N, et al. Prospective study of screening for stomach cancer in Japan. Int J Cancer 2003;106:103107.

5. Carter KJ, Schaffer HA, Ritchie WP Jr. Early gastric cancer. Ann Surg 1984;199:604-609.

6. Everett SM, Axon AT. Early gastric cancer in Europe. Gut 1997;41:142-150.

7. National cancer control programs in Korea. J Korean Med Sci 2007;22 Suppl:S3-S4.

8. Kaminski MF, Regula J, Kraszewska E, et al. Quality indicators for colonoscopy and the risk of interval cancer. N Engl J Med 2010;362:1795-1803.

9. Pabby A, Schoen RE, Weissfeld JL, et al. Analysis of colorectal cancer occurrence during surveillance colonoscopy in the dietary Polyp Prevention Trial. Gastrointest Endosc 2005;61:385-391.

10. Raftopoulos SC, Segarajasingam DS, Burke V, Ee HC, Yusoff IF. A cohort study of missed and new cancers after esophagogastroduodenoscopy. Am J Gastroenterol 2010;105:1292-1297.

11. Shiratori Y, Nakagawa S, Kikuchi A, et al. Significance of a gastric mass screening survey. Am J Gastroenterol 1985;80:831-834.

12. Mori Y, Arita T, Shimoda K, Yasuda K, Yoshida T, Kitano S. Effect of periodic endoscopy for gastric cancer on early detection and improvement of survival. Gastric Cancer 2001;4:132-136.

13. Nam SY, Choi IJ, Park KW, et al. Effect of repeated endoscopic screening on the incidence and treatment of gastric cancer in health screenees. Eur J Gastroenterol Hepatol 2009;21:855-860.

14. Lee H, Min BH, Lee JH, et al. Survival outcome associated with the screening interval for gastric cancer in Korea. Digestion 2011;84:142-148.

15. Moriguchi S, Kamakura T, Odaka T, et al. Clinical features of the differentiated and undifferentiated types of advanced gastric carcinoma: univariate and multivariate analyses. J Surg Oncol 1991;48:202-206. 
16. Adachi Y, Yasuda K, Inomata M, Sato K, Shiraishi N, Kitano S. Pathology and prognosis of gastric carcinoma: well versus poorly differentiated type. Cancer 2000;89:1418-1424.

17. Woodman CB, Threlfall AG, Boggis CR, Prior P. Is the three year breast screening interval too long? Occurrence of interval cancers in NHS breast screening programme's north western region. BMJ 1995;310:224-226.

18. Fujita S. Biology of early gastric carcinoma. Pathol Res Pract 1978;163:297-309.

19. Watanabe K, Nagata N, Shimbo T, et al. Accuracy of endoscopic diagnosis of Helicobacter pylori infection according to level of endoscopic experience and the effect of training. BMC Gastroenterol 2013;13:128.

20. Anandasabapathy S. Endoscopic imaging: emerging optical techniques for the detection of colorectal neoplasia. Curr Opin Gastroenterol 2008;24:64-69.

21. Kadish SL, Kochman ML. Endoscopic diagnosis and management of gastrointestinal malignancy. Oncology (Williston Park) 1995;9:967-983.
22. Choi KS, Jun JK, Lee HY, et al. Performance of gastric cancer screening by endoscopy testing through the National Cancer Screening Program of Korea. Cancer Sci 2011;102:1559-1564.

23. Haruma K, Kamada T, Murao T, et al. European guideline for the management of precancerous conditions in the stomach and it's application to Japan. Nihon Rinsho 2013;71:1479-1484.

24. Kapadia CR. Gastric atrophy, metaplasia, and dysplasia: a clinical perspective. J Clin Gastroenterol 2003;36:S29-S36.

25. de Vries AC, Haringsma J, de Vries RA, et al. The use of clinical, histologic, and serologic parameters to predict the intragastric extent of intestinal metaplasia: a recommendation for routine practice. Gastrointest Endosc 2009;70:18-25.

26. Correa P, Piazuelo MB, Wilson KT. Pathology of gastric intestinal metaplasia: clinical implications. Am J Gastroenterol 2010;105:493-498.

27. Kato I, Tominaga S, Ito Y, et al. Atrophic gastritis and stomach cancer risk: cross-sectional analyses. Jpn J Cancer Res 1992;83:1041-1046. 